\title{
Generation of relativistic positrons carrying intrinsic orbital angular momentum
}

\author{
Shaohu Lei®, ${ }^{1,2}$ Zhigang Bu, ${ }^{1, *}$ Weiqing Wang, ${ }^{1,2}$ Baifei Shen, ${ }^{1,3,4}$ and Liangliang $\mathrm{Ji}^{1,3, \uparrow}$ \\ ${ }^{1}$ State Key Laboratory of High Field Laser Physics, Shanghai Institute of Optics and Fine Mechanics, \\ Chinese Academy of Sciences, Shanghai 201800, China \\ ${ }^{2}$ University of Chinese Academy of Sciences, Beijing 100049, China \\ ${ }^{3}$ CAS Center for Excellence in Ultra-intense Laser Science, Shanghai 201800, China \\ ${ }^{4}$ Shanghai Normal University, Shanghai 200234, China
}

(Received 18 June 2021; accepted 20 August 2021; published 25 October 2021)

\begin{abstract}
High-energy positrons can be efficiently created through high-energy photons splitting into electronpositron pairs under the influence of the Coulomb field. Here we show that a new degree of freedom-the intrinsic orbital angular momentum (OAM) — can be introduced into relativistic positrons when the incident photons are twisted. We develop the full-twisted scattering theory to describe the transfer of angular momentum before and after the interaction. It is found that the total angular momentum (TAM) of the photon is equally distributed between the positron and electron. For each photon TAM value, the generated leptons gain a higher average OAM number when the photon spin is antiparallel to its TAM. The impact of photon polarization on the OAM spectrum profile and the scattering probability is more significant at small photon TAM numbers, owing to the various interaction channels influenced by flipping the photon spin. Our work provides the theoretical basis to study OAM physics in particle scattering and to obtain copious relativistic vortex positrons through the Beth-Heitler process.
\end{abstract}

DOI: 10.1103/PhysRevD.104.076025

\section{INTRODUCTION}

High-energy positrons are of great significance in modern particle physics experiments. Their collision with energetic electrons is essential in generating new particles such as $\mathrm{B}$ mesons/Z bosons [1,2] and monitoring various reaction processes via Bhabha scattering [3-5]. Further, in astrophysics, positrons are strongly correlated to black hole physics [6], gamma-ray bursts [7], and pair plasma physics [8,9]. Here interactions mainly concern the energy/momentum and the spin properties of the involved positrons. Recently, it was pointed out that particles can also carry intrinsic orbital angular momentum (OAM) as they do for optical photons [10-12], in the presence of vortex states [13-18]. In transmission electron microscopes (TEMs), vortex electrons have been prepared in the $80-300 \mathrm{keV}$ regime to improve the resolution and reveal new information about the subjects [19]. Interactions based on relativistic vortex particles have also been studied in the framework of quantum

\footnotetext{
zhigang.bu@siom.ac.cn

†jill@siom.ac.cn
}

Published by the American Physical Society under the terms of the Creative Commons Attribution 4.0 International license. Further distribution of this work must maintain attribution to the author(s) and the published article's title, journal citation, and DOI. Funded by SCOAP ${ }^{3}$. electrodynamics (QED), showing new features in VavilovCherenkov radiation $[20,21]$ and the possibility of creating spin-polarized particles from spinless ones [22].

Although high-energy vortex particles bring novel insights in many interactions, the generation of relativistic vortex positrons is extremely challenging. First, positrons, unlike electrons, must be created before introducing any features to the particle states. Second, manipulation of the lepton wave packets in TEMs becomes invalid in the relativistic regime, since the typical wavelengths of highenergy leptons are too small. Recalling that vortex gamma photons can be readily obtained from the Compton backscattering of Laguerre-Gaussian laser beams off highenergy electrons [23], we propose a scheme to generate vortex positrons at $\mathrm{MeV}$ energies by bombarding the vortex gamma photons onto high- $Z$ material. Relativistic electronpositron pairs can be created through the Beth-Heitler (BH) process, which facilitates efficient positron sources. By introducing a new degree of freedom-OAM-into the interaction, we obtain the law of vorticity transfer from the incident gamma photons to the created pairs. This is achieved with the first full vortex scattering theory of the $\mathrm{BH}$ process, developed in this work.

\section{THEORY OF THE VORTEX BH PROCESS}

The natural unit system $\hbar=c=1$ is applied in all calculations. We consider the process shown in Fig. 1(a). 


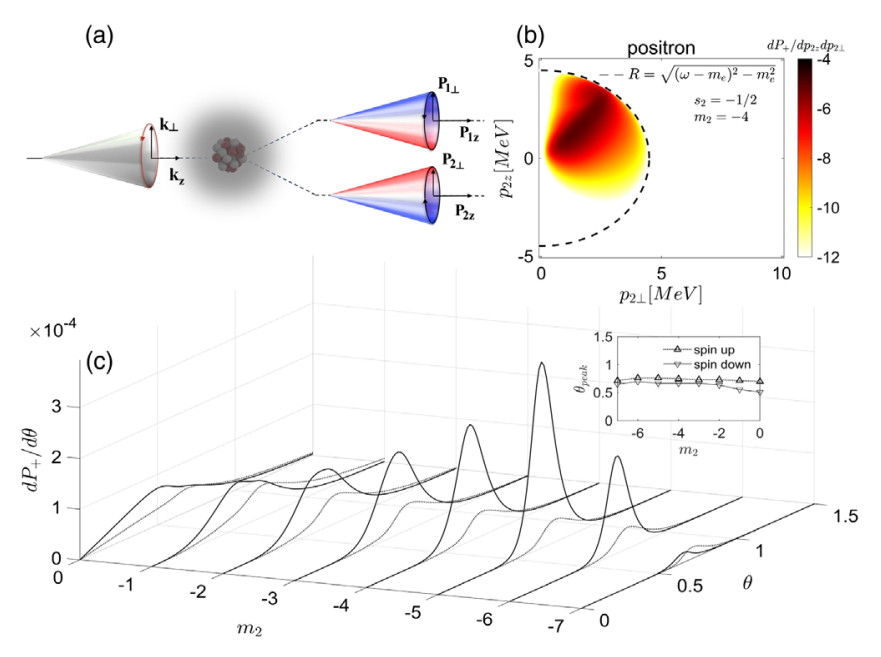

FIG. 1. (a) Schematic diagram of the twisted Beth-Heitler process where a vortex electron-positron pair is created from vortex photons bombarding high- $Z$ atoms. (b) The probability distribution of the created positron of $m_{2}=-4, s_{2}=-1 / 2$ in the momentum domain. (c) The probability distribution as a function of the opening angle at different OAM values for $s_{2}=1 / 2$ (black solid line) and $s_{2}=-1 / 2$ (black dashed line). Parameters of the incident photon are $l=6, \lambda=1, \tilde{\omega}=5 \mathrm{MeV}$.

Here, a high-energy vortex photon bombards a high- $Z$ target. Under the influence of the Coulomb field, photons may split into electron-positron pairs carrying the initial OAM information. To capture the vortex nature of the interaction, we use the Bessel modes to describe all involved particles $[18,23]$ in the cylindrical momentum space $\boldsymbol{k}^{\prime}=\left(k_{\perp}^{\prime}, \phi_{k^{\prime}}, k_{z}^{\prime}\right)$, with OAM defined with respect to the same common $z$ axis. It is a superposition of plane wave states with the same energy but different phases $\phi_{k^{\prime}}$. The twisted photon takes the form $A_{k_{\perp}, k_{z}}^{l, \lambda}(x)=$ $\int \tilde{A}_{l}\left(\boldsymbol{k}_{\perp}^{\prime}\right) A_{\boldsymbol{k}_{\perp}, k_{z}}^{l, \mu}(x) k_{\perp}^{\prime} d k^{\prime}{ }_{\perp} d \phi_{k^{\prime}}$, where $l$ is the total angular momentum (TAM) and $\lambda= \pm 1$ is the polarization parameter. Here, $A_{k}^{\lambda, \mu}(x)=1 / \sqrt{(2 \pi)^{3}(2 \omega)} \varepsilon_{k}^{\lambda, \mu} e^{-i k \cdot x}$ is the plane wave state, and $\tilde{A}_{l}\left(\boldsymbol{k}_{\perp}^{\prime}\right)=1 /\left(\sqrt{2 \pi} i^{l} k_{\perp}^{\prime}\right) \delta\left(k_{\perp}^{\prime}-k_{\perp}\right) e^{i l \phi_{k^{\prime}}}$ is the Fourier spectrum, with $\varepsilon_{\mathrm{k}}^{\lambda}$ being the polarization vector $\left(\varepsilon_{k}^{\lambda} \cdot k=0\right)$. The twisted photon field can be expressed as

$$
\begin{aligned}
& A_{k_{\perp}, k_{z}}^{l, \lambda ; \mu}(x)=\varepsilon_{k_{\perp}, k_{z}}^{l, \lambda ; \mu}(\boldsymbol{r}) e^{i k_{z} z-i \omega t}=\frac{e^{i k_{z} z-i \omega t}}{4 \pi \sqrt{\omega}} \\
& \quad \times\left(\begin{array}{c}
(i / 2)\left[\left(1-k_{z} / \omega\right) \Theta_{k_{\perp}}^{l+\lambda}(\boldsymbol{r})+\left(1+k_{z} / \omega\right) \Theta_{k_{\perp}}^{l-\lambda}(\boldsymbol{r})\right] \\
(\lambda / 2)\left[\left(1-k_{z} / \omega\right) \Theta_{k_{\perp}}^{l+\lambda}(\boldsymbol{r})-\left(1+k_{z} / \omega\right) \Theta_{k_{\perp}}^{l-\lambda}(\boldsymbol{r})\right] \\
\left(\lambda k_{\perp} / \omega\right) \Theta_{k_{\perp}}^{l}(\boldsymbol{r})
\end{array}\right),
\end{aligned}
$$

where $\omega$ is the photon energy, $\Theta_{k_{\perp}}^{n}(\boldsymbol{r})=J_{n}\left(k_{\perp} r\right) e^{i n \theta}$ is the transverse function, and $J_{n}(r)$ is the Bessel function of the first kind. The Coulomb field takes the form $A_{0}^{\operatorname{Coul}}(\vec{x})=-Z e /|\vec{x}|=-4 \pi Z e \int 1 /(2 \pi)^{3} e^{-\vec{q} \cdot \vec{x}} /|\vec{q}|^{2} d^{3} q$. We notice that the vortex photon field is not equivalent to the eigenmodes of the OAM operator $\hat{L}_{z}=-i \partial / \partial \theta$ defined along the $z$ direction. For weak spin-orbital coupling, the OAM value can be well represented by the quantity $l-\lambda$.

The twisted electron and positron states can be constructed from the positive- and negative-frequency plane wave solutions of the Dirac equation $\psi_{p}^{+, s}(x)=$ $1 / \sqrt{2(2 \pi)^{3}} e^{-i p \cdot x}\left(\sqrt{1+M / E} \xi^{s}, \sqrt{1-M / E}(\boldsymbol{\sigma} \cdot \boldsymbol{\kappa}) \xi^{s}\right)^{\mathrm{T}}$ and $\quad \psi_{p}^{-, s}(x)=1 / \sqrt{2(2 \pi)^{3}} e^{i p \cdot x}\left(\sqrt{1-M / E}(\boldsymbol{\sigma} \cdot \boldsymbol{\kappa}) \eta^{s}\right.$, $\left.\sqrt{1+M / E} \eta^{s}\right)^{\mathrm{T}}$, where $\boldsymbol{\kappa}=\boldsymbol{p} /|\boldsymbol{p}|$ is the unit vector of the momentum, while $\xi^{s}$ and $\eta^{s}$ are the two-component spinors characterizing the electron and positron spins in the rest frame. The Fourier spectrum is the same as that of photons. The one for positrons is therefore [18]

$$
\begin{aligned}
\psi_{p_{\perp}, p_{z}}^{-, m, s}(x)= & v_{p_{\perp}, p_{z}}^{m, s}(r, \theta) e^{-i p_{z} z+i E t}=\frac{e^{-i p_{z} z+i E t}}{\sqrt{2}(2 \pi)} \\
& \times\left[\left(\begin{array}{c}
\sqrt{1-\frac{M}{E} p_{z}|\boldsymbol{p}|} \sigma^{3} \eta^{s} \\
\sqrt{1+\frac{M}{E}} \eta^{s}
\end{array}\right) \Theta_{p_{\perp}}^{m}(\boldsymbol{r})\right. \\
& \left.-\frac{i p_{\perp}}{|\mathrm{p}|} \sqrt{1-\frac{M}{E}}\left(\begin{array}{c}
\sigma_{m, p_{\perp}}^{\perp}(r, \theta) \eta^{s} \\
0
\end{array}\right)\right]
\end{aligned}
$$

with $\sigma_{p_{\perp}}^{\perp, m}(r, \theta)=\left(\begin{array}{cc}0 & -\Theta_{p_{\perp}}^{m-1}(\mathrm{r}) \\ \Theta_{p_{\perp}}^{m+1}(\mathrm{r}) & 0\end{array}\right)$. The wave function of the twisted electron is similar, with a different plane wave bispinor, $u_{p_{\perp}, p_{z}}^{m, s}(r, \theta)$. Here, $m$ stands for the OAM number and $s$ for the spin number. In principle, the OAM number is not the eigenvalue of the vortex wave function. It is, however, a good approximation if the spinorbital interaction (SOI) is not significant. This is the case for the parameters considered here; hence, we take $m$ as the OAM in the following and discuss the effect of SOI in later sections. In the perturbation theory, the scattering matrix of twisted photons splitting into positron-electron pairs is $S_{f i}=S_{1}+S_{2}$. The first term is

$$
\begin{aligned}
S_{1}= & -\frac{i e^{2}}{2} \int d^{4} x d^{4} x^{\prime} \frac{d^{4} q}{(2 \pi)^{4}} \bar{\psi}_{p_{1 \perp}, p_{1 z}}^{+, m_{1}, s_{1}}(x) \mathcal{A}_{k_{\perp}, k_{z}}^{l, \lambda}(x) \\
& \times \frac{(\not 1+M)}{\left(q^{2}-M^{2}\right)} e^{-i q \cdot\left(x-x^{\prime}\right)} \gamma^{0} \psi_{p_{2 \perp}, p_{2 z}}^{-, m_{2}, s_{2}}\left(x^{\prime}\right) A_{0}^{\mathrm{Coul}}\left(x^{\prime}\right) .
\end{aligned}
$$

Here, $M$ is the mass of the electron/positron, and $\mathscr{A}=A_{\mu} \gamma^{\mu}$. Substituting the twisted photon state from Eq. (1) and the Coulomb field and electron-positron states from Eq. (2) into Eq. (3), we get 


$$
\begin{aligned}
S_{1}= & \frac{i Z e^{3}}{4(2 \pi)} \sqrt{\frac{\left(E_{1}-M\right)\left(E_{2}-M\right)}{\omega E_{1} E_{2}}} \frac{1}{\left|\mathrm{p}_{1}\right|\left|\mathrm{p}_{2}\right|} \delta\left(\omega-E_{1}-E_{2}\right) \\
& \times \xi^{s_{1} \dagger} \Xi_{k_{\perp}, k_{z}}^{l, \lambda}\left(m_{1}, p_{1 \perp}, p_{1 z} ; m_{2}, p_{2 \perp}, p_{2 z}\right) \eta_{\substack{s_{2} \\
s_{E_{q}=-E_{2}=E_{1}-\omega} \\
q_{z}=p_{1 z}-k_{z}}},
\end{aligned}
$$

with

$$
\Xi_{k_{\perp}, k_{z}}^{l, \lambda}=\left(\begin{array}{cc}
\varsigma_{11} \delta_{l, m_{1}-m_{2}} & \varsigma_{12} \delta_{l, m_{1}-m_{2}+1} \\
\varsigma_{21} \delta_{l, m_{1}-m_{2}-1} & \varsigma_{22} \delta_{l, m_{1}-m_{2}}
\end{array}\right) .
$$

The integer $l$ represents the photon TAM number, while $m_{1}$ and $m_{2}$ are the OAM numbers of electrons and positrons, respectively. Details of the matrix elements $\varsigma_{11}, \varsigma_{12}, \varsigma_{21}$, and $\varsigma_{22}$ are included in the Appendix. The matrix $S_{2}$ is obtained by exchanging the photon and the Coulomb field in $S_{1}$, which leads to a different matrix $\tilde{\Xi}$ (see the Appendix). Each matrix element in $\Xi$ and $\tilde{\Xi}$ determines the creation probability of pairs with different spin polarizations, and the angular momentum (AM)-dependent Kronecker delta function gives the corresponding selection rule for the twisted $\mathrm{BH}$ process:

$$
l=m_{1}-m_{2}+\Delta,
$$

with $\Delta=0, \pm 1$. The minus sign before $m_{2}$ in Eq. (6) is consistent with the definition of positron AM. To calculate the creation probability, we use the wave packet to describe the incident photon $\mathcal{A}_{\mu}^{\lambda, l}(x)=\int 1 / \sqrt{2 \omega} \rho\left(k_{\perp}, k_{z}\right) \times$ $A_{k_{\perp}, k_{z} ; \mu}^{\lambda, l}(x) d k_{z} d k_{\perp}$, where the weighting function taking Gaussian distributions $\rho\left(k_{\perp}, k_{z}\right)=N_{\tau_{\perp}, \tau_{z}} \exp \left[-\left(k_{\perp}-\tilde{k}_{\perp}\right)^{2} /\right.$ $\left.\tau_{\perp}^{2}-\left(k_{z}-\tilde{k}_{z}\right)^{2} / \tau_{z}^{2}\right]$, with central momenta and energy $\tilde{k}_{\perp}, \tilde{k}_{z}$ and $\tilde{\omega}$. The wave-packet widths in the transverse and longitudinal directions are $\tau_{\perp}$ and $\tau_{z}$. Under the narrow wave-packet approximation $\tau_{\perp(z)} \ll \tilde{k}_{\perp(z)}$, we get

$$
\begin{aligned}
\left|S_{\text {wave-packet }}\right|^{2}= & C \times Z^{2} \pi \alpha^{3} \delta\left(\tilde{\omega}-E_{1}-E_{2}\right) \\
& \times \frac{\left(E_{1}-M\right)\left(E_{2}-M\right)}{\tilde{\omega} E_{1} E_{2}\left|\boldsymbol{p}_{1}\right|^{2}\left|\boldsymbol{p}_{2}\right|^{2}} \\
& \times \operatorname{Tr}\left[\xi^{s_{1}} \xi^{s_{1} \dagger}(\Xi+\tilde{\Xi}) \eta^{s_{2}} \eta^{s_{2} \dagger}(\Xi+\tilde{\Xi})^{\dagger}\right],
\end{aligned}
$$

where

$$
\begin{aligned}
C & =\frac{N_{\tau_{\perp}, \tau_{z}}^{2}}{2} \exp \left(-\frac{2 \tilde{\omega}^{2}}{\tau^{2}}\right) \frac{\sqrt{\pi} \tau}{2 \sqrt{2}} \int d \theta_{k^{\prime}} d \theta_{k} \sin \theta_{k^{\prime}} \sin \theta_{k} \\
& \times\left[1+\operatorname{Erf}\left(\frac{1}{\sqrt{2} \tau}\left(\tilde{k}_{\perp}\left(\sin \theta_{k^{\prime}}+\sin \theta_{k}\right)+\tilde{k}_{z}\left(\cos \theta_{k^{\prime}}+\cos \theta_{k}\right)\right)\right)\right] \\
& \times \exp \left[\frac{1}{2 \tau^{2}}\left(\tilde{k}_{\perp}\left(\sin \theta_{k^{\prime}}+\sin \theta_{k}\right)+\tilde{k}_{z}\left(\cos \theta_{k^{\prime}}+\cos \theta_{k}\right)\right)^{2}\right] \tilde{\omega}^{3}
\end{aligned}
$$

is a coefficient associated with the wave packet, and $\theta$ is the opening angle of the incident particle in the wave packet, $\tan (\theta) \equiv k_{\perp} / k_{z}$. The pair creation probability is

$$
d \mathcal{P}=p_{1 \perp} p_{2 \perp}\left|S_{\text {wave-packet }}\right|^{2} d p_{1 \perp} d p_{1 z} d p_{2 \perp} d p_{2 z} .
$$

\section{RESULTS}

In order to calculate the scattering probabilities, we set the central energy and momenta of the photon wave packet as $\tilde{\omega}=5 \mathrm{MeV}, \tilde{k}_{z}=4 \mathrm{MeV}, \tilde{k}_{\perp}=3 \mathrm{MeV}$; the photon polarization is $\lambda=1$, and the TAM number is $l=6$. We consider photons interacting with copper atoms $(Z=29)$. By integrating the momentum of the electron in Eq. (9), we obtain the creation probability of the positron. As is known in plane wave scattering, the interaction follows energy and momentum conservation, leading to a thin resonance line in the momentum space, as represented by the dashed circle in Fig. 1(b). However, in vortex scattering, the conservation is for energy alone. The resonance condition is significantly relaxed, such that the probability is distributed in a broad region in Fig. 1(b). Furthermore, it is seen that the probability peaks along a certain angle. In Fig. 1(c), we summarize the angular-dependent distribution of positrons at different OAM values $m_{2}$. While the highest value varies with the OAM, all profiles exhibit peaks at similar angles. The average angular distribution is around $\theta \approx 0.68$, which coincides with the azimuthal angle of the incident photon, $\tan (\theta)=k_{\perp} / k_{z}=3 / 4, \theta=0.64$. In other words, the azimuthal angle at which most positrons are created is largely conserved in a paraxial collision. One should notice that in plane wave scattering, the scattering angle is dependent on the outgoing particle energy.

We also compare the distributions of the final positron with spin-up and spin-down states in Fig. 1(c). For the case we considered, the spin-down channel contributes to a large part of the interaction probability. Thus, the generated particle is obviously polarized. This is consistent with the propagation of polarization from polarized photons to positrons [24-26]. The polarization is, however, not $100 \%$, indicating spin-orbital coupling during the scattering process.

A central question about the vortex scattering is how OAM is distributed among the final particles. In Fig. 2, we show OAM spectra for the generated pair and their relationship with the TAM of the initial photon. We integrate the momentum and sum over the spins of created pairs to get the total probabilities at different OAM numbers, as shown in Fig. 2(a) with photon TAM values of $l=5,10$, 15,20 . The electron primarily carries OAM with the same sign as the photon, while that of the positron is opposite. This is because the defined direction of positron OAM is opposite to that of an electron. As the photon TAM increases, the central OAM shifts towards large values accordingly. Furthermore, the total creation probability 

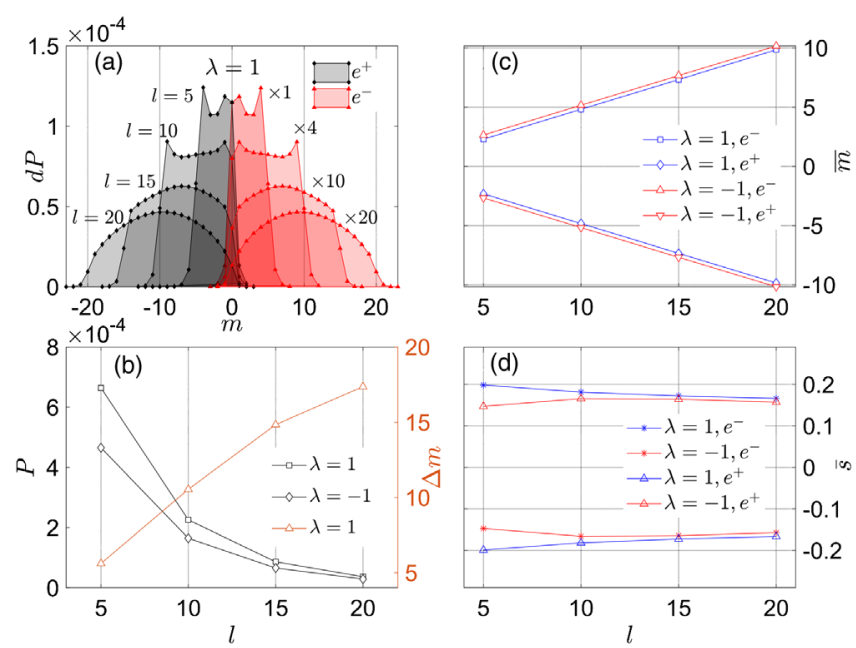

FIG. 2. (a) The OAM spectra of generated positrons (gray) and electrons (red) corresponding to the photon TAM numbers of $5,10,15$, and 20. (b) The total creation probability and the OAM spectrum width as a function of the photon TAM. (c),(d) The average OAM and spin values of the electron-positron pair in the cases where the photon TAM is (c) parallel to polarization $(\lambda=1)$ and (d) antiparallel to polarization $(\lambda=-1)$.

declines, and the OAM spectrum width increases at larger photon TAM values, as illustrated in Fig. 2(b).

Figure 2(c) shows the averaged OAM number of the created electron and positron after summing over the lepton spins. It is seen that the electron and positron gain equal average OAM, which are $\bar{m}_{1}=-\bar{m}_{2}=2.302$ (2.646), 4.820 (5.165), 7.330 (7.666), and 9.836 (10.160) for $\lambda=$ $1(-1)$ with $l=5,10,15$, and 20 , respectively, and the corresponding equal average SAM values are $\bar{s}_{1}=-\bar{s}_{2}=$ $0.199(-0.147), 0.181(-0.166), 0.172(-0.164)$, and $0.166(-0.157)$. In either case, the symmetry among the electron and positron is preserved: $\bar{m}_{1}+\bar{s}_{1}=-\bar{m}_{2}-$ $\bar{s}_{2}=l / 2$, and the partition of OAM satisfies the average TAM conservation: $\bar{m}_{1}-\bar{m}_{2}+\bar{s}_{1}-\bar{s}_{2}=l$.

A flip of the photon polarization also changes the orientation of lepton spins. It is seen in Fig. 2(d) that the averaged spin number turns over when switching from $\lambda=1$ to $\lambda=-1$, which leads to higher average OAM in Fig. 2(c) (red line). In fact, the effect of photon spin is also imprinted in the asymmetric OAM distributions in Fig. 2(a) at relatively small photon TAM numbers, e.g., $l=5$ and 10. This effect becomes less significant when $l$ is large. We show the total scattering probability as a function of the photon TAM number in Fig. 2(c) with different photon spins. It is seen that at each TAM value, the probability is notably higher for $\lambda=1$. The difference is much more suppressed when increasing the TAM.

To reveal how photon polarization affects the distribution of positron and electron OAM, we divide the interaction into four channels determined by the final spin states of the leptons and compare their OAM spectra in Fig. 3 with those of the photon TAM values $l=10$ and 20. First of all, for

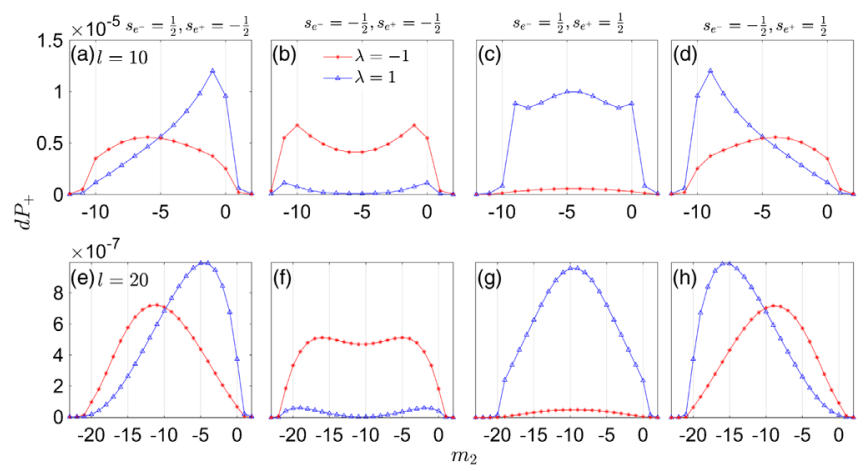

FIG. 3. Four channels of the OAM spectrum for positrons when the photon polarization is varied between $\lambda=1$ (blue) and $\lambda=-1$ (red). Here, the photon TAM is (a)-(d) $l=10$ and (e)-(h) $l=20$.

$\lambda=1$ we find that the $(-1 / 2,-1 / 2)$ channel in Figs. 3(b) and 3(f) is significantly suppressed as compared to the $(1 / 2,1 / 2)$ channel in Figs. 3(c) and 3(g). The situation is reversed when switching to the $\lambda=-1$ case. The trends presented here are consistent with the polarized scattering of plane wave states. The channels shown in Figs. 3(b) $(-1 / 2,-1 / 2)$ and 3 (c) $(1 / 2,1 / 2)$ are independently symmetrical, exhibiting centers at $m_{2}=-5.5$ and $m_{2}=-4.5$ $(l=10, \lambda=1)$. This is related to the $\delta$ functions in Eq. (5), which are responsible for the channels in Figs. 3(b) $\delta_{l, m_{1}-m_{2}-1}$ and 3(c) $\delta_{l, m_{1}-m_{2}+1}$, where there is one unit shift for both the positron and electron. We see that the distribution centers do not necessarily correspond to the spectrum peaks. In fact, the two probability peaks are located at $m_{2}=-10$ and -1 in Fig. 2(b), corresponding to $m_{1}=1$ and 10 for the electron (not shown here), respectively. Therefore, the combinations of electron-positron OAM values $(1,-10)$ and $(10,-1)$ dominate the channel. Adding the other channels leads to the nonmonopole profiles in Fig. 2(a) at $l=10$.

Flipping the photon spin to $\lambda=-1$ induces large variations to the profiles of the channels shown in Figs. 3(a) and $3(d)$, showing an enormous shift of the peak positrons. The distributions indicate that $\left(m_{1}, m_{2}\right)=(9,-1)$ and $(1,-9)$ are primary. We sum the two distributions (both have $s_{1}+s_{2}=0$ ) to produce symmetrical spectra centered at $m_{2}=-5$ for each photon spin state. As a result, the major difference in terms of average OAM stems from changing the spectrum center from the channel in Fig. 3(c) $(\lambda=1)$ to that in Fig. 3(b) $(\lambda=-1)$. This is why the leptons gain a slightly larger OAM in the latter case.

For $l=20$, the spectra of Figs. 3(f) and 3(g) are much more smooth and monopole-like. After summing up the four channels, the OAM distribution shows good symmetry, as seen in Fig. 2(a). This is because the ratio between photon spin and TAM is much smaller here. As mentioned above, a large disparity in the scattering probability between different photon spins is found in Fig. 2(b). 


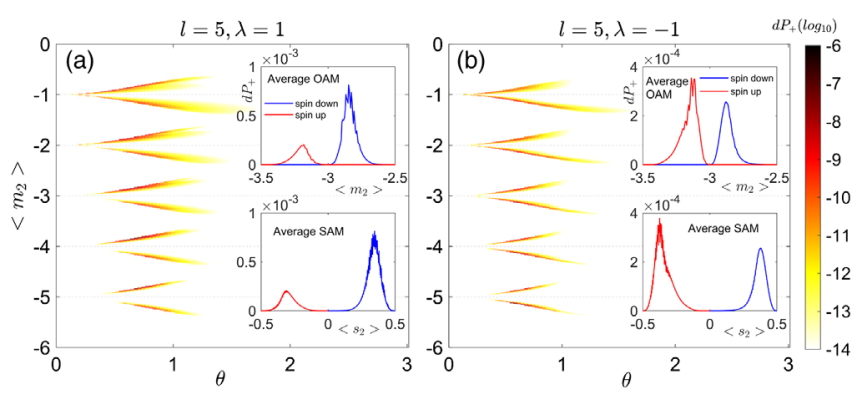

FIG. 4. Dependence of the scattering probability on the average OAM and opening angle of positron with (a) $l=5, \lambda=1$ and (b) $l=5, \lambda=-1$ when considering the spin-orbital coupling during interactions. Inner figures show the average OAM and spin after integrating the opening angle, indicating the deviation from the quantum numbers $m_{2}=-3$ and $s_{2}= \pm 1 / 2$.

We notice that at $l=10$, the quantity related to the photon OAM is approximately $l-\lambda=9$ for $\lambda=1$ and $l-\lambda=11$ for $\lambda=-1$. To make a comparison at a commensurate OAM value, one should choose $l=8$ for $\lambda=-1$ rather than $l=10$. We have the scattering probabilities of $P=$ $2.26 \times 10^{-4}$ with $(l=10, \lambda=1, l-\lambda=9)$ and $P=2.4 \times$ $10^{-4}$ with $(l=8, \lambda=-1, l-\lambda=9)$. In other words, the difference with the same $l-\lambda$ vanishes. This observation suggests that the photon spin has little effect on the total probability when keeping $l-\lambda$ (rather than $l$ ) constant.

\section{DISCUSSION}

Spin-orbit coupling naturally exists in the quantum vortex state in the relativistic regime. The average OAM and spin angular momentum (SAM) of the vortex positron state after considering the SOI are [18]

$$
\langle m\rangle=\hbar(m+\Delta s) \hat{z}, \quad\langle s\rangle=\hbar(s-\Delta s) \hat{z}
$$

Here, $\Delta=(1-M / E) \sin ^{2} \theta$ denotes the SOI-induced change. We take the photon TAM $l=5$ with different polarizations to calculate the possibility as a function of the average OAM and SAM in Fig. 4. As the opening angle $\theta$ increases, both the average OAM $\langle m\rangle$ and SAM $\langle s\rangle$ deviate from the quantized integrals. In general, the peak position of average SAM is slightly lower than $|s|=1 / 2$ in all cases, and the one for $\langle m\rangle$ is also quite close to the OAM quantum number. These results validate our approximations in the analysis.

\section{CONCLUSIONS}

We employed the twisted states of photons and electrons/ positrons to calculate the scattering probabilities of twisted photons into twisted electron-positron pairs under the influence of the Coulomb field. We found that the photon TAM is efficiently transferred to the final leptons. We discussed the effect of spin-orbit coupling on average OAM and SAM and noticed that angular momentum is also transferred from spin to orbit. This work lays the foundation of generating relativistic vortex positron beams for new physics in a number of twisted scattering processes.

\section{ACKNOWLEDGMENTS}

This work is supported by the National Science Foundation of China (Grants No. 11875307 and No. 11935008), the Strategic Priority Research Program of the Chinese Academy of Sciences (Grant No. XDB16010000), and the Ministry of Science and Technology of the People's Republic of China (Grants No. 2018YFA0404803).

\section{APPENDIX: DERIVATION OF THE VORTEX BH PROCESS}

The scattering matrix of this process is

$$
\begin{aligned}
\mathcal{M}_{f i}= & -\frac{e^{2}}{2} \int d^{4} x d^{4} x^{\prime} \frac{d^{4} q}{(2 \pi)^{4}} \bar{u}_{p_{1 \perp}, p_{1 z}}^{m_{1}, s_{1}}(x) e^{-i p_{1 z} z+i E_{1} t} \gamma^{\mu} A_{k_{1 \perp}, k_{1}, \mu}^{l_{1}, \lambda_{1}}(x) \frac{i(\not 1+M)}{\left(q^{2}-M^{2}\right)} e^{-i q \cdot\left(x-x^{\prime}\right)} \gamma^{0} v_{p_{2 \perp}, p_{2 z}}^{m_{2}, s_{2}}\left(r^{\prime}, \theta^{\prime}\right) e^{-i p_{2 z} z^{\prime}+i E_{2} t^{\prime}} A_{0}^{\mathrm{Coul}}\left(x^{\prime}\right) \\
& -\frac{e^{2}}{2} \int d^{4} x d^{4} x^{\prime} \frac{d^{4} q}{(2 \pi)^{4}} \bar{u}_{p_{1 \perp}, p_{1 z}}^{m_{1}, s_{1}}(x) e^{-i p_{1 z} z+i E_{1} t} \gamma^{0} A_{0}^{\operatorname{Coul}}(x) \frac{i(\not q+M)}{\left(q^{2}-M^{2}\right)} e^{-i q \cdot\left(x-x^{\prime}\right)} \gamma^{\nu} v_{p_{2}, p_{2 z}}^{m_{2}, s_{2}}\left(r^{\prime}, \theta^{\prime}\right) e^{-i p_{2 z} z^{\prime}+i E_{2} t^{\prime}} A_{k_{1 \perp}, k_{1 z}, \nu}^{l_{1}, \lambda_{1}}\left(x^{\prime}\right)
\end{aligned}
$$

Calculate the two items separately the first item Eq. (4) becomes:

$$
\begin{aligned}
\mathcal{M}_{1, f i}= & -\frac{i Z e^{3}}{4(2 \pi)} \sqrt{\frac{\left(E_{1}-M\right)\left(E_{2}-M\right)}{\omega E_{1} E_{2}}} \frac{1}{\left|\mathrm{p}_{1} \| \mathrm{p}_{2}\right|} \delta\left(\omega-E_{1}-E_{2}\right) \\
& \times \xi^{s_{1} \dagger}\left(\mathrm{I}_{1}+\mathrm{II}_{1}+\mathrm{III}_{1}+\mathrm{IV}_{1}+\mathrm{V}_{1}+\mathrm{VI}_{1}+\mathrm{VII}_{1}+\mathrm{VIII}_{1}+\mathrm{VIIII}_{1}\right) \eta_{\substack{s_{2} \\
\sum_{q}=-E_{2}=E_{1}-\omega \\
q_{z}=p_{1 z}-k_{1 z}}}
\end{aligned}
$$

$\mathrm{I}_{1}-\mathrm{VIIII}_{1}$ are all $2 \times 2$ matrices through integration, and we find that the items at the corresponding positions have the same delta function. Taking $I_{1}$ as an example, the ultimate result is 


$$
\begin{aligned}
\mathrm{I}_{1} & =p_{1 z} p_{2 z}\left(E_{q}+M\right) \int \frac{d q_{\perp}^{\prime} q_{\perp}^{\prime}}{\left(p_{1 z}+p_{2 z}-k_{1 z}\right)^{2}+q_{\perp}^{\prime 2}} \frac{d q_{\perp} q_{\perp}}{\left(E_{q}^{2}-\left(p_{1 z}-k_{1 z}\right)^{2}-q_{\perp}^{2}-M^{2}\right)} \\
& \times\left(\begin{array}{c}
\frac{\lambda_{1} k_{1 \perp}}{\omega_{1}} S_{l_{1}}^{m_{1}}\left(k_{1 \perp}, p_{1 \perp}, q_{\perp}\right) S_{m_{2}}^{m_{2}}\left(q_{\perp}, p_{2 \perp}, q_{\perp}^{\prime}\right) \delta_{m_{2}, m_{1}-l_{1}}-i\left(1+\frac{\lambda_{1} k_{1 z}}{\omega_{1}}\right) S_{l_{1}-1}^{m_{1}}\left(k_{1 \perp}, p_{1 \perp}, q_{\perp}\right) S_{m_{2}}^{m_{2}}\left(q_{\perp}, p_{2 \perp}, q_{\perp}^{\prime}\right) \delta_{m_{2}, m_{1}-l_{1}+1} \\
-i\left(1-\frac{\lambda_{1} k_{1 z}}{\omega_{1}}\right) S_{l_{1}+1}^{m_{1}}\left(k_{1 \perp}, p_{1 \perp}, q_{\perp}\right) S_{m_{2}}^{m_{2}}\left(q_{\perp}, p_{2 \perp}, q_{\perp}^{\prime}\right) \delta_{m_{2}, m_{1}-l_{1}-1} \quad-\frac{\lambda_{1} k_{1 \perp}}{\omega_{1}} S_{l_{1}}^{m_{1}}\left(k_{1 \perp}, p_{1 \perp}, q_{\perp}\right) S_{m_{2}}^{m_{2}}\left(q_{\perp}, p_{2 \perp}, q_{\perp}^{\prime}\right) \delta_{m_{2}, m_{1}-l_{1}}
\end{array}\right) .
\end{aligned}
$$

Among them, we define the integral of the triple Bessel product:

$$
\begin{aligned}
S_{n}^{m}(p, k, q) & =\int d r r J_{n}(p r) J_{m-n}(q r) J_{m}(k r)=(-1)^{m} \int d r r J_{n}(p r) J_{m-n}(q r) J_{-m}(k r) \\
& =\frac{(-1)^{m} \delta}{2 \pi A_{p, q, k}} \cos \left(n\left(\pi-\angle_{p, q}\right)+m\left(\pi-\angle_{k, q}\right)\right)=\frac{(-1)^{n} \delta}{2 \pi A_{p, q, k}} \cos \left(n \angle_{p, q}+m \angle_{k, q}\right) \\
& =\frac{(-1)^{m+n} \delta}{2 \pi A_{p, q, k}} \cos \left(m \angle_{p, k}+(m-n) \angle_{p, q}\right)=\frac{\delta}{2 \pi A_{p, q, k}} \cos \left(n \angle_{p, k}-(m-n) \angle_{k, q}\right)
\end{aligned}
$$

$\mathrm{I}_{1}-\mathrm{VIIII}_{1}$ are all similar to $\mathrm{I}_{1}$; each matrix element is the product of two $S_{n}^{m}(p, k, q)$ functions (with different $m$ and $n$ coefficients) and is multiplied by the corresponding coefficients that depend on the photon momentum energy and polarization.

$\mathcal{M}_{2, f i}$ is calculated in the same way, and finally we can get

$\mathcal{M}_{f i}=\mathcal{M}_{1, f i}+\mathcal{M}_{2, f i}=\frac{i Z e^{3}}{4(2 \pi)} \sqrt{\frac{\left(E_{1}-M\right)\left(E_{2}-M\right)}{\omega E_{1} E_{2}}} \frac{1}{\left|\mathrm{p}_{1}\right|\left|\mathrm{p}_{2}\right|} \delta\left(\omega-E_{1}-E_{2}\right) \xi^{s_{1} \dagger}\left(\left.\Xi\right|_{\substack{E_{q}=-E_{2}=E_{1}-\omega \\ q_{z}=p_{1 z}-k_{1 z}}}+\tilde{\Xi}_{\substack{\tilde{\Xi} \\ \sum_{q}=E_{1}=\omega-E_{2} \\ q_{z}=k_{1 z}-p_{2 z}}}\right) \eta^{s_{2}}$

[1] J. Haba, Nucl. Instrum. Methods Phys. Res., Sect. A 368, 74 (1995).

[2] S. Roth, Precision Electroweak Physics at Electron-Positron Colliders (Springer, Berlin; New York, 2007).

[3] F. Ambrosino, A. Antonelli, Antonelli et al., Eur. Phys. J. C 47, 589 (2006).

[4] C. M. Carloni Calame, C. Lunardini, G. Montagna, O. Nicrosini, and F. Piccinini, Nucl. Phys. B584, 459 (2000).

[5] W. Beenakker, F. A. Berends, and S. C. van der Marck, Nucl. Phys. B349, 323 (1991).

[6] D. C. Homan, R. Ojha, D. H. Roberts, and J. Wardle, Nature (London) 395, 457 (1998).

[7] P. Mészáros, Annu. Rev. Astron. Astrophys. 40, 137 (2002).

[8] E. G. Blackman and G. B. Field, Phys. Rev. Lett. 71, 3481 (1993).

[9] H. A. Weldon, Phys. Rev. Lett. 66, 293 (1991).

[10] L. Allen, M. W. Beijersbergen, R. Spreeuw, and J. P. Woerdman, Phys. Rev. A 45, 8185 (1992).

[11] M. J. Padgett, Opt. Express 25, 11265 (2017).

[12] N. B. Simpson, K. Dholakia, L. Allen, and M. J. Padgett, Opt. Lett. 22, 52 (1997).

[13] K. Y. Bliokh, Y. P. Bliokh, S. Savelev, and F. Nori, Phys. Rev. Lett. 99, 190404 (2007).

[14] J. Verbeeck, H. Tian, and P. Schattschneider, Nature (London) 467, 301 (2010).
[15] M. Uchida and A. Tonomura, Nature (London) 464, 737 (2010).

[16] B. J. McMorran, A. Agrawal, Anderson et al., Science 331, 192 (2011).

[17] C. Zhang, P. Xu, and X. Jiang, AIP Adv. 10, 105230 (2020).

[18] K. Y. Bliokh, M. R. Dennis, and F. Nori, Phys. Rev. Lett. 107, 174802 (2011).

[19] F. Tamburini, G. Anzolin, G. Umbriaco, A. Bianchini, and C. Barbieri, Phys. Rev. Lett. 97, 163903 (2006).

[20] I. P. Ivanov, V. G. Serbo, and V. A. Zaytsev, Phys. Rev. A 93, 053825 (2016).

[21] I. Kaminer, M. Mutzafi, A. Levy et al., Phys. Rev. X 6, 011006 (2016).

[22] I. P. Ivanov, N. Korchagin, A. Pimikov, and P. Zhang, Phys. Rev. Lett. 124, 192001 (2020).

[23] U. D. Jentschura and V. G. Serbo, Phys. Rev. Lett. 106, 013001 (2011).

[24] T. Omori, M. Fukuda, T. Hirose et al., Phys. Rev. Lett. 96, 114801 (2006).

[25] G. Alexander, J. Barley, Y. Batygin et al., Phys. Rev. Lett. 100, 210801 (2008).

[26] D. Abbott, P. Adderley, A. Adeyemi et al., Phys. Rev. Lett. 116, 214801 (2016). 